\title{
Mutation analysis in 46 British and Irish patients with Gaucher's disease
}

\author{
C E Hatton, A Cooper, C Whitehouse, J E Wraith
}

\begin{abstract}
DNA from 46 unrelated patients with Gaucher's disease was analysed for 10 known mutations: 84GG(c84 G 85ins), N370S (c1226G), L444P (c1448C), R463C (c1504T), R496H (c1604A), IVS2+1, D409H (c1342C), RecNciI (c1448C-1498C), RecTL (c1342C-1498C), and c1263del (c12641318del). Fifty four mutations $(90 \%)$ were identified in 30 patients with type I disease. These included a previously undescribed recombinant mutation RecA456P (c1448C-1484C). Thirteen (54\%) of 24 type II alleles were identified, including one new point mutation $\mathrm{N} 462 \mathrm{~K}$ (c1503G) and one new 55bp deletion also incorporating the RecTL mutations c1263del+RecTL (c1264del-1498C). All four type III patients were homozygous for the L444P point mutation. Generally, patients with one copy of the N370S mutation had mild adult onset disease, regardless of the nature of their second mutation. Three exceptions had childhood onset disease and genotypes N370S/ R463C, N370S/RecA456P, and N370S/?. The L444P/L444P genotype was thought to be associated with neurological disease. Two type I patients with this genotype who exhibited no central nervous system disease were identified, however. The R463C and c1263del mutations were found to be present at a higher frequency than reported in other populations and they should be included in any mutation screen of this population. The recombinant mutations RecA456P and c1263del+RecTL have not been previously described and are the fourth and fifth recombinant mutations identified in the glucocerebrosidase gene.
\end{abstract}

(Arch Dis Child 1997;77:17-22)

Willink Biochemical Genetics Unit, Royal Manchester Children's Hospital

C E Hatton

A Cooper

C Whitehouse

J E Wraith

Correspondence to: Dr Alan Cooper, Willink Biochemical Genetics Unit, Royal Manchester Children's Hospital, Hospital Road Pendlebury, Manchester M27 4HA.

Accepted 18 March 1997

Gaucher's disease is the most often encountered lysosomal storage disease. ${ }^{1}$ It is caused by a drastic reduction in glucocerebrosidase activity that results in the lysosomal storage of glucocerebroside. The autosomal recessive disorder is clinically heterogeneous and has historically been divided into three subtypes. Type I is characterised by a lack of central nervous system disease; symptoms may include hepatosplenomegaly, anaemia, pancytopenia, and progressive bone disease. Onset may be in childhood or adulthood and in the mildest forms patients may be clinically asymptomatic. Type II presents in infancy with gross neurological deterioration, as well as the symptoms of type I, and early death. Type III is a less acute form with childhood onset and more slowly progressive neurological disease. The variable phenotype is probably related to different mutations in the functional glucocerebrosidase gene, but molecular analysis has often shown a poor correlation between genotype and phenotype and it is highly likely that another factor or factors contributes to the phenotypic presentation. ${ }^{2}$ Type I disease is present at a high frequency in Ashkenazi Jews, ${ }^{3}$ whereas type III is common in Norbottnian Swedes. ${ }^{4}$

We describe mutation analysis in 46 British and Irish patients to illustrate the different mutation frequency in this population compared with that well documented in other countries.

\section{Patients and methods}

The diagnosis of Gaucher's disease was initially made by the assay of $\beta$ glucosidase in white blood cells, or in one case by an assay of uncultured chorionic villi. ${ }^{5}$

Genomic DNA from 46 unrelated patients with Gaucher's disease was isolated from peripheral blood leucocytes, cultured skin fibroblasts, or homogenised chorionic villi by boiling in $50 \mathrm{mmol} / \mathrm{l} \mathrm{NaOH}$ followed by neutralisation with $1 \mathrm{~mol} / 1 \mathrm{TRIS} / \mathrm{HCl} \mathrm{pH} 7.5$.

Screening for IVS2+1, N370S, D409H, L444P, R463C, and R496H point mutations was performed by polymerase chain reaction (PCR) amplification and restriction enzyme digestion. ${ }^{3}$ Screening for the $84 \mathrm{GG}$ insertion was performed by the amplification refraction mutation system (ARMS) ${ }^{6}$ using wild type and mutant specific primers. Table 1 gives the primers, annealing temperatures, and restriction enzymes used.

All primers used were positioned in regions the glucocerebrosidase gene where the closely related pseudogene sequence was absent or non-homologous so that only the active gene was amplified. D409H, L444P, and R463C 5' primers and the N370S 3' primer were all positioned within the genomic region $5878-5932$, a 55 bp region in exon 9 , which is deleted in the pseudogene. Genomic numbering is as described by Horowitz et al. ${ }^{7}$ cDNA nucleotide numbers begin with the $\mathrm{A}$ of the 
Table 1 PCR and sequencing primers

\begin{tabular}{|c|c|c|c|c|c|}
\hline $\begin{array}{l}\text { cDNA No } \\
\text { (amino acid) }\end{array}$ & Primer genomic No & Primer sequence & $\begin{array}{l}\text { Annealing } \\
\text { temperature }\end{array}$ & Restriction site & Reference No \\
\hline IVS $2+1$ & $\begin{array}{l}979-997 \\
1316-1335\end{array}$ & $\begin{array}{l}\text { GAATGTCCCAAGCCTTTGA } \\
\text { AGCTGAAGCAAGAGAATCGC }\end{array}$ & $61^{\circ} \mathrm{C}$ & $-\mathrm{Hph} 1$ & 3 \\
\hline c1226G & $5807-5826$ & GCCTTTGCTCTTACCCTCGA & $61^{\circ} \mathrm{C}$ & +Xho1 & $\mathrm{NP}$ \\
\hline (N370S) & 5906-5926 & ACGAAAGTTACGCACCCAATT & & & \\
\hline c1342C & $5878-5897$ & GCTTGCCCTGAACCCCGAAG & $58^{\circ} \mathrm{C}$ & -Sty1 & NP \\
\hline$(\mathrm{D} 409 \mathrm{H})$ & $6031-6050$ & GAGATGATAGGCCTGGTATG & & & \\
\hline c1448C & $5904-5923$ & CCAATTGGGTGCGTAACTTT & $61^{\circ} \mathrm{C}$ & +Ncil & NP \\
\hline (L444P) & $6655-6691$ & CTCACTGGCGACGCCACAGGTAGGTGTGAATGGAGTA & & & \\
\hline $\mathrm{c} 1504 \mathrm{~T}$ & $5904-5923$ & CCAATTGGGTGCGTAACTTT & $61^{\circ} \mathrm{C}$ & - Msp1 & $\mathrm{NP}$ \\
\hline (R463C) & $6655-6691$ & CTCACTGGCGACGCCACAGGTAGGTGTGAATGGAGTA & & & \\
\hline c1604A & $6632-6651$ & TCCTGGAGACAATCTCACCT & $61^{\circ} \mathrm{C}$ & $+\mathrm{Hph} 1$ & 3 \\
\hline (R496H) & $6782-6801$ & TAAGCTCACACTGGCCCTGC & & & \\
\hline c84 G 85 ins & $\mathrm{N}: 1007-1036$ & N:GCATCATGGCTGGCAGCCTCACAGGATTGC & $70^{\circ} \mathrm{C}$ & & NP \\
\hline 84GG & M:1007-1036 & M:GCATCATGGCTGGCAGCCTCACAGGATTGG & & & \\
\hline $9-11$ & $\begin{array}{l}5569-5588 \\
7314-7333\end{array}$ & $\begin{array}{l}\text { AACCATGATTCCCTATCTTC } \\
\text { GGTTTTTCTACTCTCATGCA }\end{array}$ & $50^{\circ} \mathrm{C}$ & & 10 \\
\hline 9 & $\begin{array}{l}5797-5816 \\
6782-6801\end{array}$ & $\begin{array}{l}\text { CCAGTGTTGAGCCTTTGTCT } \\
\text { GAGATGATAGGCCTGGTATG }\end{array}$ & $58^{\circ} \mathrm{C}$ & & 10 \\
\hline 10 & $\begin{array}{l}6322-6341 \\
6542-6561\end{array}$ & $\begin{array}{l}\text { GGTTTCATGGGAGGTACCCC } \\
\text { GAGAGTGTGATCCTGCCAAG }\end{array}$ & $58^{\circ} \mathrm{C}$ & & $\mathrm{NP}$ \\
\hline
\end{tabular}

$\mathrm{NP}=$ new primer.

Genomic nucleotide numbering is according to Horowitz et al. ${ }^{7} \mathrm{cDNA}$ numbering begins with the A of the upstream initiator codon. The reference sequence for the cDNA numbering is M11080.

upstream initiator ATG. ${ }^{2}$ Polymerase chain reaction amplification of exon 9 was performed on patients apparently homozygous for these mutations and also on all patients with unknown mutations. Sequencing of exons 9 and 10 was performed on patients heterozygous or homozygous for the L444P mutation to detect the complex recombinant alleles RecNciI (containing point mutations L444P, A456P (c1484C) and V460V (1498C)) and RecTL (containing point mutations $\mathrm{D} 409 \mathrm{H}$, L444P, A456P and V460V), formed by crossing over between the active gene and pseudogene or by gene conversion. ${ }^{89}$ This also distinguishes between L444P and L444R (c1448G) mutations, ${ }^{2}$ which is not possible by digestion with NciI. The presence of all R463C mutations was confirmed by sequencing exon 10 to distinguish them from the adjacent $\mathrm{R} 463 \mathrm{H}$ $(\mathrm{c} 1505 \mathrm{~A})^{2}$ and new N462K mutation, identified in this study.

Sequencing of both strands of exons 9 and 10 was carried out in a two stage amplification using the primers shown in table $1 .^{10}$ The second stage PCR products of exons 9 and 10 were purified using the QIAquick PCR purification kit (QIAGen) and sequenced using ABI Prism dye terminator cycle sequencing ready reaction kits containing Amplitaq DNA polymerase, FS (Perkin Elmer) and analysed on an ABI Prism 377 DNA sequencer. The resultant sequences were imported into Sequence Navigator (Perkin Elmer) for alignment, editing, and mutation analysis. Samples found to have a deletion in exon 9 after PCR were analysed by excising individual DNA fragments from $2 \%$ agarose gel and purifying using the QIAquick gel extraction kit (QIAGen) before sequencing.

Results and discussion

Initial screening for the IVS2+1, N370S, $\mathrm{D} 409 \mathrm{H}, \mathrm{L} 444 \mathrm{P}, \mathrm{R} 463 \mathrm{C}$, and $\mathrm{D} 496 \mathrm{H}$ mutations was performed by digestion with the appropriate restriction enzymes after amplification using PCR and functional gene specific primers (table 1). This method alone is unreliable for the N370S, L444P, and R463C mutations. Firstly, mutations adjacent to L444P and R463C create or destroy restriction sites for NciI and MspI, preventing accurate identification of these mutations by restriction digestion analysis. Hence it is essential to confirm the presence of these mutations by sequencing. One patient, apparently heterozygous for R463C, was subsequently shown to carry a previously undescribed mutation $\mathrm{N} 462 \mathrm{~K}$ by automated sequencing. This mutation is a $\mathrm{C} \rightarrow \mathrm{G}$ transversion at $\mathrm{cDNA} 1503$. It disrupts the MspI recognition site CCGG, as does R463C. Secondly, the five prime primers for $\mathrm{N} 370 \mathrm{~S}$ L444P and R463C are located in a position where the pseudogene has a $55 \mathrm{bp}$ deletion. This deletion, however, is also known to occur in the functional gene $(\mathrm{c} 1263 \mathrm{del})^{10}$ and, if present, would result in a false homozygous result for any of the above mutations. To overcome this problem it is essential that the c1263del should be screened for. This can be done by PCR amplification of exons 9 to 11 . The amplification products are then reamplified using nested primers spanning exon 9 . In patients with the $55 \mathrm{bp}$ deletion, three bands were seen after electrophoresis, one band with increased mobility and one with decreased mobility compared with the single band seen in normal subjects (data not shown). The presence of three bands was at first surprising; however, after excision from the gel the three bands were sequenced using fluorescent dye terminator cycle sequencing. As expected, the smallest band was shown to contain the $55 \mathrm{bp}$ deletion at genomic position 5878-5932 (fig 1B), whereas the least mobile band was found to be a heteroduplex of the normal and c1263del alleles (data not shown). Its reduced mobility in agarose gels probably resulted from the unpaired 55bp loop retarding migration. False homozygosity for L444P (one patient L444P/c1263del+RecTL) and R463C (one patient $\mathrm{R} 463 \mathrm{C} / \mathrm{c} 1263 \mathrm{del}$ ) was seen using restriction digestion and the presence of the 


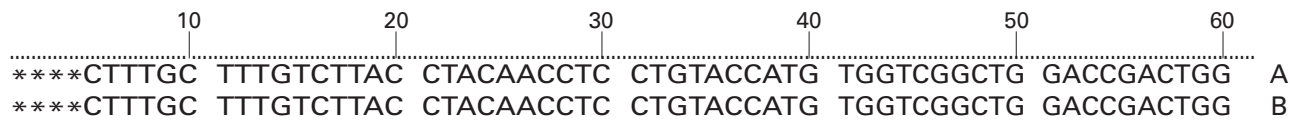

\begin{tabular}{|c|c|c|c|c|c|}
\hline 70 & 80 & 90 & 100 & 110 & 120 \\
\hline AACCTTGCCC & TGAACCCCGA & AGGAGGACCC & AATTGGGTGC & GTAACTTTGT & CGACAGTCCC \\
\hline AA $\ldots \ldots$ & $\ldots \ldots$ & $\ldots \ldots$ & $\ldots \ldots$ & $\ldots$ & $\ldots-\ldots-$ CCC \\
\hline 130 & 140 & 150 & 160 & 170 & 180 \\
\hline
\end{tabular}

Figure 1 Sequence Navigator aligned sequence of part of exon 9 of the glucocerebrosidase gene illustrating a 55bp deletion. (A) Normal sequence and (B) sequence of a patient with the c1263del mutation. The sequencing of deleted alleles was performed using template excised and eluted from agarose gels after electrophoretic separation.

c1263del allele was later shown by amplification of exon 9 and sequencing as described earlier. Misgenotyping due to the presence of a deletion has been reported by Beutler et al, who identified a patient erroneously diagnosed as homozygous N370S, but actually N370S/total gene del. ${ }^{11}$

The L444P mutation can arise by one of two mechanisms. The first is as a simple point mutation. This mutation occurs naturally, however, in the pseudogene sequence and can be incorporated into the functional gene by crossover events. Two such events have been reported, designated $\mathrm{RecNciI}^{7}$ and RecTL. ${ }^{9}$ The former results in the incorporation of three mutations naturally occurring in the pseudogene sequence into the functional gene, L444P, A456P, and V460V. Crossover in the RecTL mutation incorporates a fourth mutation present in the pseudogene $\mathrm{D} 409 \mathrm{H}$. This mutation may also be present due to a simple point mutation in some subjects. ${ }^{12}$ All patients found to have the L444P mutation were further investigated for recombinant mutations by automated dye terminator cycle sequencing, those with RecNciI by sequencing exon 10, and those with RecTL by sequencing exons 9 and 10 after identification of the $\mathrm{D} 409 \mathrm{H}$ mutation by restriction analysis. Figure $2 \mathrm{~A}$ and $2 \mathrm{~B}$ show the presence of the three mutations L444P (in the homozygous state) and A456P and V460V (in the heterozygous state) in a patient with the genotype L444P/RecNciI compared with a normal sequence.

In this study, using these techniques, we identified two more unique recombinant events in this region of the glucocerebrosidase gene. One mutation includes both the four RecTL mutations and the c1263del deletion. Sequencing of exon 10 revealed the L444P, A456P, and V460V mutations (fig 3A), whereas sequencing of the smaller exon 9 band excised from agarose gel showed the presence of c1263del and $\mathrm{D} 409 \mathrm{H}$ (fig 4). This mutation appears to have arisen by a recombination between the active gene and the pseudogene occurring

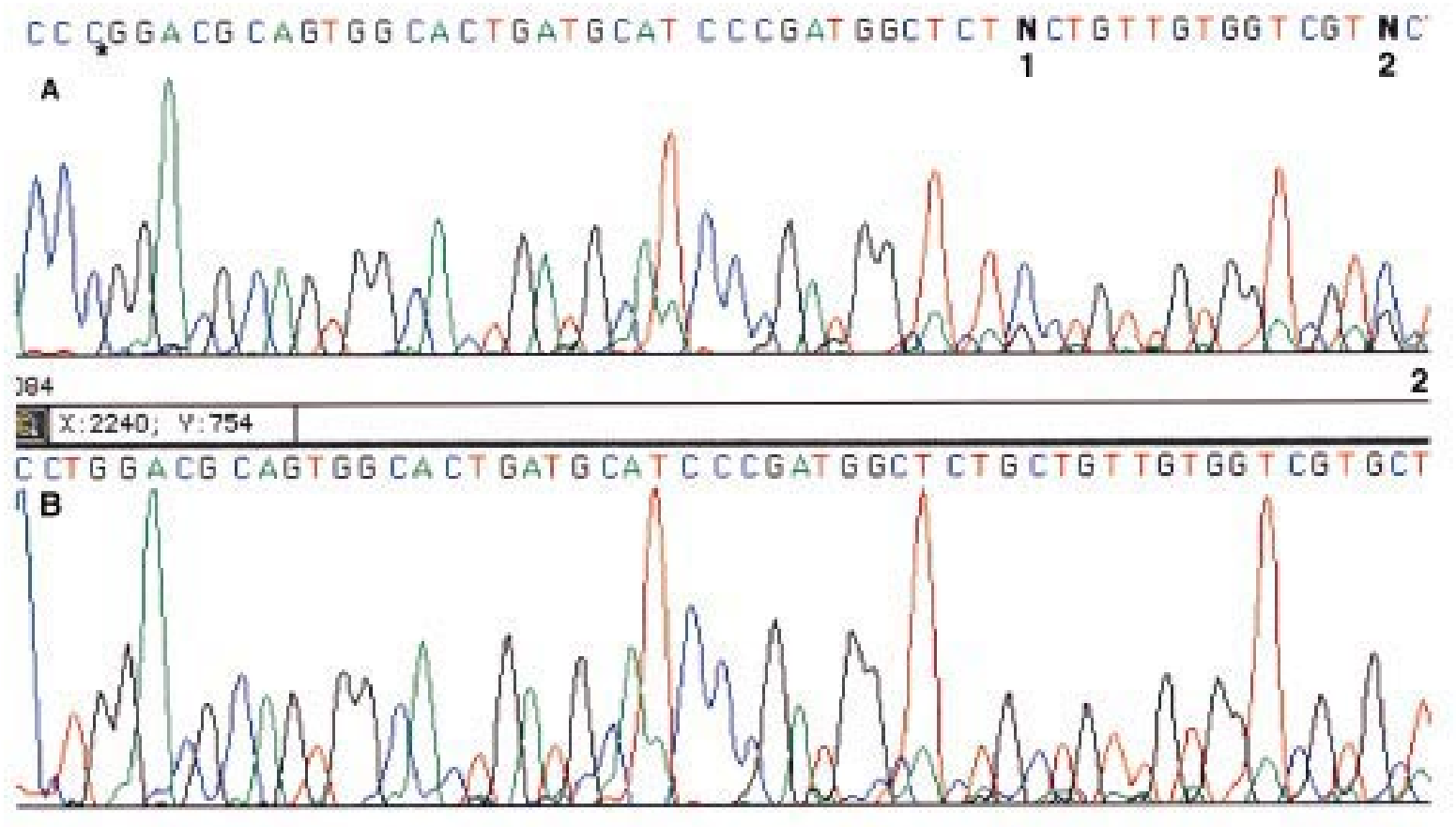

Figure 2 Electrophoretogram of dye terminator sequencing of part of exon 10. (A) Patient heterozygous for RecNciI and L444P and (B) normal control. $\star, L 444 P$ mutation $(T \rightarrow C)$; N1, A456P mutation $(G \rightarrow C)$; and N2, V460V mutation $(G \rightarrow C)$. 

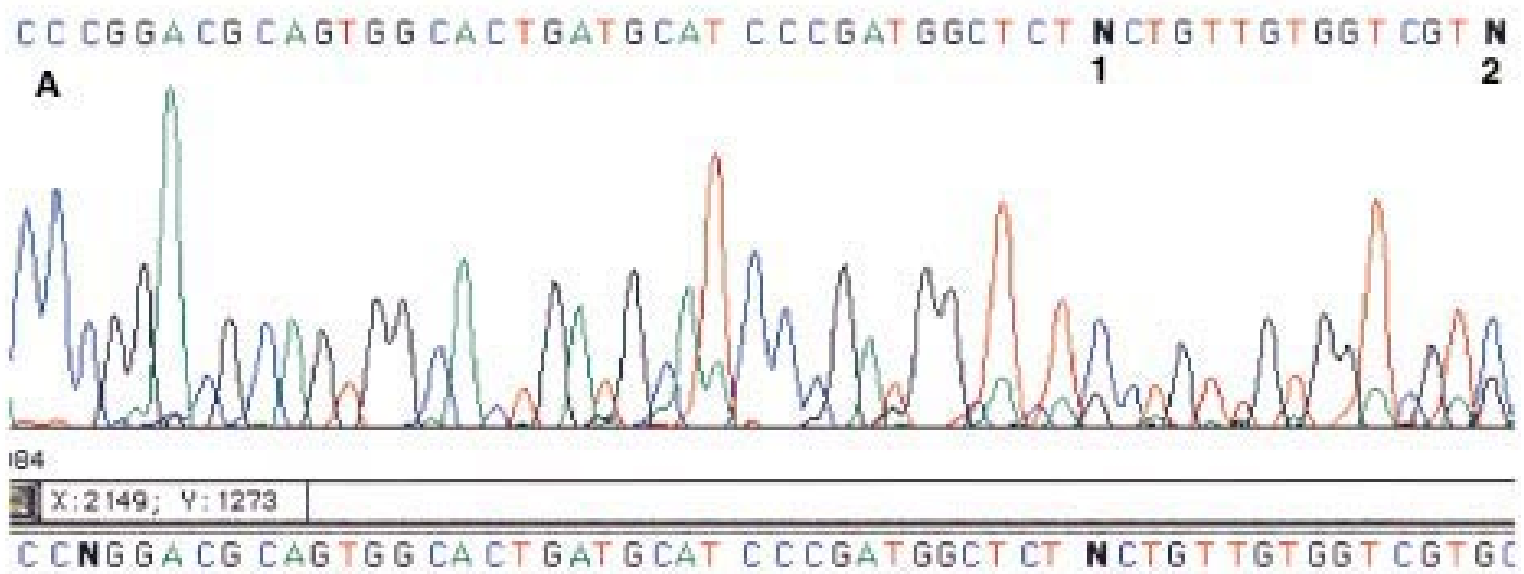

B

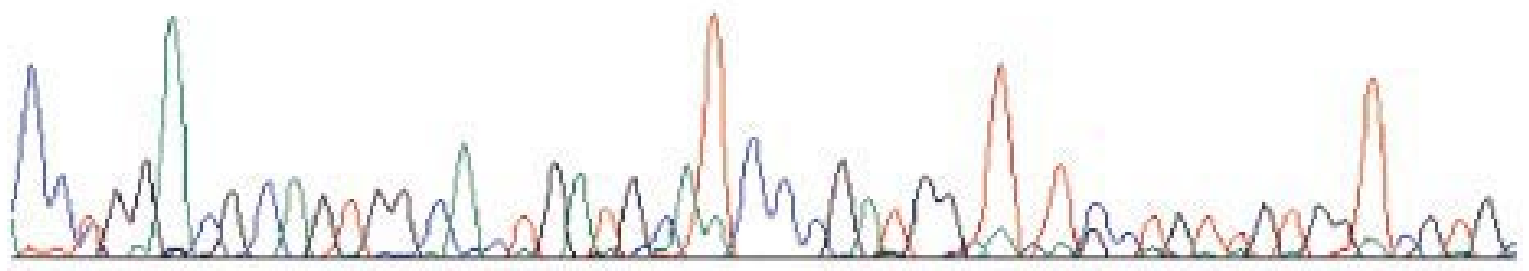

Figure 3 Electrophoretogram of dye terminator sequencing of part of exon 10. (A) Patient heterozygous for L444P and c1263del + RecTL mutations and (B) patient heterozygous for N370S and RecA456P mutations. N1, A456P mutation $(G \rightarrow C) ; N 2$, V460V mutation (G $\rightarrow C$ ).

between genomic 5588-5877 and 6483-7314. As a result of the great homology between the pseudo and functional genes, the exact break points could not be determined. It has been designated c1263del+RecTL and was combined with an L444P point mutation in the one patient identified. The second recombinant mutation introduces both the L444P and A456P mutations into the functional sequence, but the V460V mutation is not involved (fig 3B). This mutation has been designated RecA456P and appears to be due to recombination between the active and pseudogene occurring between genomic 5957-6272 and 6469-6481, as the $\mathrm{C} \rightarrow \mathrm{T}$ difference normally present in the pseudogene was identified at position 6272 of the functional gene by sequencing. The $\mathrm{D} 409 \mathrm{H}$ mutation at the end of exon 9 was also shown to be absent (data not shown). Identification of these new crossover events brings to five the number of recombinant mutations so far described in the glucocerebrosidase gene. ${ }^{2}$

The distribution of the 10 mutations screened for and new mutations identified is shown in tables 2 and 3 (mutations 84GG,
$\mathrm{R} 496 \mathrm{H}$, and RecTL were not detected). All mutations were identified in the four Ashkenazi Jewish patients, two being homozygous N370S and the other two heterozygous N370S (N370S/RecNciI and N370S/R463C). The distribution of mutations in this limited population correlates well with that found by other studies of Ashkenazi Jewish patients. ${ }^{313}$ The N370S mutation has been reported to be associated with mild non-neurological disease. ${ }^{14}$ All four of our patients homozygous for N370S have adult onset type I disease. Complete genotyping has clarified the prognosis for these patients, who may be considered for enzyme replacement treatment, and has allowed carrier detection for family members. This, however, has created counselling dilemmas with the identification of genetically affected (N370S/ N370S and N370S/R463C), but clinically normal, siblings. ${ }^{15}$

Of the 24 non-Jewish patients of British and Irish origin with type I Gaucher's disease, 89\% of mutations were identified, the three most common being N370S (29\%), L444P (25\%), and R463C (21\%) (table 2). With three exceptions patients heterozygous for the N370S

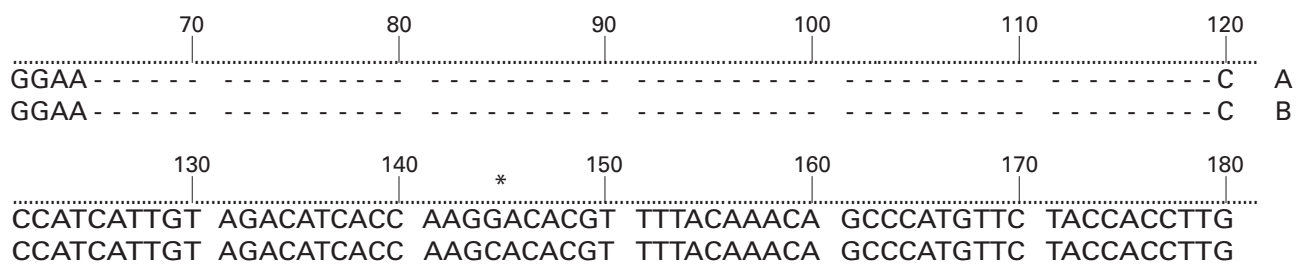

Figure 4 Sequence Navigator aligned sequence of part of exon 9 of the glucocerebrosidase gene illustrating $(A)$ a patient heterozygous for the c1263del mutation and (B) a patient heterozygous for the c1263del+RecTL mutation. The D409H recombinant mutation $(G \rightarrow C)$ is starred. Sequencing of deleted alleles was performed using template excised and eluted from agarose gels after electrophoretic separation. 
Table 2 Frequency of the mutations studied in the three clinical types of Gaucher's disease

\begin{tabular}{|c|c|c|c|c|c|c|c|c|c|c|c|}
\hline & Unknown & N370S & $L 444 P$ & $R 463 C$ & $I V S 2+1$ & D409H & $N 462 K$ & c1263del & $\operatorname{RecNciI}$ & $\operatorname{Rec} A 456 P$ & $c 1263 \mathrm{del}+\operatorname{RecTL}$ \\
\hline Jewish alleles & \multirow{2}{*}{\multicolumn{3}{|c|}{$6(75)$}} & \multirow{2}{*}{\multicolumn{2}{|c|}{$1(12.5)$}} & & & & \multirow{2}{*}{\multicolumn{2}{|c|}{$1(12.5)$}} & \\
\hline Type I & & & & & & & & & & & \\
\hline \multicolumn{12}{|l|}{ Non-Jewish alleles, } \\
\hline Type I & $6(11)$ & $15(29)$ & $13(25)$ & $11(21)$ & $1(2)$ & $1(2)$ & & $1(2)$ & $3(6)$ & $1(2)$ & \\
\hline Type II & $11(46)$ & & $10(42)$ & & & & $1(4)$ & $1(4)$ & & & $1(4)$ \\
\hline Type III & & & $8(100)$ & & & & & & & & \\
\hline Total non-Jewish & $17(20)$ & $15(18)$ & $31(37)$ & $12(13)$ & $1(1.2)$ & $1(1.2)$ & $1(1.2)$ & $2(2.4)$ & $3(3.6)$ & $1(1.2)$ & $1(1.2)$ \\
\hline
\end{tabular}

Numbers in parentheses are percentage of total alleles.

mutation had mild adult onset disease regardless of the nature of their other mutation. The exceptions had childhood onset disease and genotypes N370S/?, N370S/RecA456P, and N370S/R463C. Interestingly, the N370S/ $\mathrm{R} 463 \mathrm{C}$ genotype was also seen in a mild adult onset case of the disease (table 3 ). Nine other patients with type I disease had childhood onset disease. Two of these had the genotype L444P/L444P, once thought to be consistently associated with severe disease and central nervous system involvement. ${ }^{14}$ Later reports support our finding that this genotype may also result in non-neurological disease. ${ }^{216}{ }^{17}$ The elder of our two patients is 30 years old and has no neurological disease, although she has severe bone disease.

The R463C mutation is more prevalent in this population than reported in other nonJewish populations ${ }^{14}$ and has probably been underreported. Only one study of the UK population has included this mutation. ${ }^{18}{ }^{19} \mathrm{We}$ found one adult patient with type I disease with homozygous $\mathrm{R} 463 \mathrm{C}$ using both restriction enzyme digestion and sequencing. This is the first patient identified to be homozygous for this mutation. ${ }^{2}$ The patient had a mild adult onset phenotype, suggesting that $\mathrm{R} 463 \mathrm{C}$ is a relatively mild mutation.

All four patients with type III disease were homozygous L444P. Homozygosity for this mutation is associated with type III disease in Norbottnian Swedes ${ }^{4}$; however, this correlation between genotype and phenotype cannot be applied to other populations.

Of the 12 patients with type II disease, only $54 \%$ of mutations were identified, most (42\%) being heterozygous L444P/unknown. The high proportion of unknown mutations in patients

Table 3 Genotype to phenotype correlation of the mutations detected

\begin{tabular}{lllll}
\hline Genotype & Type I adult & Type I childhood & Type II & Type III \\
\hline N370S/N370S & $4(2)$ & 0 & 0 & 0 \\
N370S/L444P & 1 & 0 & 0 & 0 \\
N370S/R463C & $4(1)$ & 1 & 0 & 0 \\
N370S/RecNciI & $2(1)$ & 0 & 0 & 0 \\
N370S/RecA456P & 0 & 1 & 0 & 0 \\
N370S/? & 3 & 1 & 0 & 0 \\
L444P/L444P & 0 & 2 & 1 & 4 \\
L444P/R463C & 2 & 1 & 0 & 0 \\
L444P/c1263del+RecTL & 0 & 0 & 1 & 0 \\
L444P/? & 1 & 1 & 7 & 0 \\
R463C/R463C & 1 & 0 & 0 & 0 \\
R463C/RecNciI & 0 & 2 & 0 & 0 \\
R463C/IVS2+1 & 0 & 1 & 0 & 0 \\
R463C/D409H & 0 & 1 & 0 & 0 \\
R463C/c1263del & 0 & 1 & 0 & 0 \\
c1263del/? & 0 & 0 & 1 & 0 \\
N462K/? & 0 & 0 & 1 & 0 \\
?? & 0 & 0 & 1 & 0
\end{tabular}

Numbers in parentheses are patients of Ashkenazi Jewish origin. with type II disease makes carrier testing of family members and their partners problematic. Only two patients had a complete genotype, one being homozygous L444P and one L444P/c1263del+RecTL.

The c1263del deletion in exon 9 has been previously reported to be rare, ${ }^{20}$ but may be more prevalent in the British and Irish population than elsewhere. As its presence can cause erroneous classification of other genotypes it should be included in any mutation screening programme. Our study has shown that, except for the N370S mutation, the correlation between genotype and phenotype is poor in British and Irish patients with Gaucher's disease and other factors must contribute to clinical presentation. Complex recombinant alleles, deletions, and new mutations were found in a significant proportion of patients, indicating that detailed mutation analysis using sequencing is required to accurately genotype these patients to understand the implications for phenotype and to provide reliable carrier detection within the family.

We thank Mrs Jackie Gardner for preparing sequencing gels

1 Beutler E, Grabowski GA. Gaucher disease. In: Scriver CE, Beaudet AL, Sly WS, Valle D, eds. The metabolic and molecular basis of inherited disease. 7th Ed. New York: molecular basis of inherited dise.

2 Beutler E, Gelbart T. Glucocerebrosidase (Gaucher disease). Hum Mutat 1996;8:207-13.

3 Beutler E, Gelbart T, Kuhl W, Zimran A, West C. Mutations in Jewish patients with Gaucher disease. Blood 1992;79: 1662-6.

4 Dahl N, Langerstrom M, Erikson A, Petterson U. Gaucher disease type III (Norrbottnian type) is caused by a single mutation in exon 10 of the glucocerebrosidase gene. Am $\mathcal{F}$ Hum Genet 1990;47:1589-90.

5 Peters SP, Lee RE, Glew RH. A microassay for Gaucher disease. Clin Chim Acta 1975;60:391-6.

6 Newton CR, Graham A, Heptinstall LE. Analysis of any point mutation in DNA. The amplification refactory mutation system (ARMS). Nucleic Acids Res 1989;17:2503-15.

7 Horowitz M, Wilder S, Horowitz Z, Reiner O, Gelbart T, Beutler E. The human glucocerebrosidase gene and pseudogene: structure and evolution. Genomics 1989;4:87pseudo.

8 Zimran A, Sorge J, Kubitz M, West C, Beutler E. A glucocerebrosidase fusion gene in Gaucher disease. Implications for the molecular anatomy, pathogenesis and diagcations for the molecular anatomy, pathogenesis and
nosis of this disorder. $\mathcal{F}$ Clin Invest 1990;85:219-22.

9 Latham T, Grabowski GA, Theophilus BD, Smith FI. Complex alleles of the acid $\beta$-glucocerebrosidase genes. Am f Hum Genet 1990;45:79-86.

10 Beutler E, Gelbart T, West C. Identification of six new Gaucher disease mutations. Genomics 1993;15:203-5.

11 Beutler E, Gelbart T. Erroneous assignment of Gaucher disease genotype as a consequence of a complete gene deletion. Hum Mutat 1995;4:212-6.

12 Abrahamov A, Elstein D, Gross-Tsur V, et al. Gaucher's disease variant characterised by progressive calcification of heart valves and unique genotype. Lancet 1995;346:10002.

13 Horowitz M, Tzuri G, Eyal N, et al. Prevalence of nine mutations among Jewish and non-Jewish Gaucher disease mutations among Jewish and non-Jewish Gau

14 Zimran A, Gross E, West C, Sorge J, Kubitz M, Beutler E. Prediction of severity of Gaucher disease by identification of mutations at DNA level. Lancet 1989;ii:349-52. 
15 Zimran A, Gelbart T, Westwood B, Grabowski GA, Beutler E. High frequency of the 1226 mutation for type I Gaucher disease among the Ashkenazi Jewish population. Am f Hum Genet 1991;49:855

16 Kawame H, Maekawa K, Eto Y. Molecular screening of Japanese patients with Gaucher disease: phenotypic variability in the same genotypes. Hum Mutat 1993;2:362 7

17 Sidransky E, Bottler A, Stubblefield B, Ginns E. DNA mutational analysis of type 1 and type 3 Gaucher patients. How well do mutations predict phenotype? Hum Mutat
1994;3:25-8.

18 Walley AJ, Barth ML, Ellis I, Fensom AH, Harris A. Gaucher's disease screening in the United Kingdom: screening non-Jewish patients for the two common mutations. F Med Genet 1993;30:280-3.

19 Mistry PK, Cox T. The glucocerebrosidase locus in Gaucher's disease: molecular analysis of a lysosomal enzyme. F Med Genet 1993;30:889-94.

20 Beutler E. Gaucher disease: new molecular approaches to diagnosis and treatment. Science 1992;256:794-9. 